\title{
Collapse of magnetized hypermassive neutron stars in general relativity
}

\author{
Matthew D. Duez ${ }^{1}$ Y Yuk Tung Liu ${ }^{1}$, Stuart L. Shapiro ${ }^{1}{ }^{1}$ Masaru Shibata ${ }^{2}$, and Branson C. Stephens ${ }^{1}$ \\ ${ }^{1}$ Department of Physics, University of Illinois at Urbana-Champaign, Urbana, IL 61801, USA \\ ${ }^{2}$ Graduate School of Arts and Sciences, University of Tokyo, Komaba, Meguro, Tokyo 153-8902, Japan
}

\begin{abstract}
Hypermassive neutron stars (HMNSs) - equilibrium configurations supported against collapse by rapid differential rotation - are possible transient remnants of binary neutron star mergers. Using newly developed codes for magnetohydrodynamic simulations in dynamical spacetimes, we are able to track the evolution of a magnetized HMNS in full general relativity for the first time. We find that secular angular momentum transport due to magnetic braking and the magnetorotational instability results in the collapse of an HMNS to a rotating black hole, accompanied by a gravitational wave burst. The nascent black hole is surrounded by a hot, massive torus undergoing quasistationary accretion and a collimated magnetic field. This scenario suggests that HMNS collapse is a possible candidate for the central engine of short gamma-ray bursts.
\end{abstract}

PACS numbers: 04.25.Dm, 04.30.-w, 04.40.Dg

Hypermassive neutron stars (HMNSs) figure prominently in several relativistic astrophysical systems of current interest. Mass limits for nonrotating stars [the Oppenheimer-Volkoff (OV) limit] and for rigidly rotating stars (the supramassive limit, which is only about $20 \%$ larger) can be significantly exceeded by the presence of differential rotation [1]. Stars with masses greater than the supramassive limit are called hypermassive stars. Mergers of binary neutron stars (BNSs) can lead to the formation of HMNS remnants. This possibility was explored in Newtonian and post-Newtonian simulations [2, 3], and in full general relativity [4]. The latest relativistic BNS merger simulations with realistic equations of state (EOS) [5] confirm that HMNS formation is indeed a possible outcome. HMNSs can also result from core collapse of rotating massive stars, since rapid differential rotation can develop during the collapse.

Differentially rotating stars approach rigid rotation via transport of angular momentum on secular time scales. HMNSs, however, cannot settle down to rigidly rotating equilibria since their masses exceed the maximum allowed by uniform rotation. Thus, 'delayed' collapse to a black hole, and possibly mass loss, will follow transport of angular momentum from the inner to the outer regions. Previous calculations of HMNS collapse have focused on viscous angular momentum transport $[\underline{6}]$ and angular momentum loss due to gravitational radiation [5]. In this Letter, we demonstrate black hole formation induced by seed magnetic fields in HMNSs.

In any highly conducting astrophysical plasma, a frozen-in magnetic field can be amplified appreciably by gas compression or shear (e.g. differential rotation). Even when an initial seed magnetic field is weak, the field can grow to influence significantly the system dynamics.

\footnotetext{
${ }^{*}$ Currently address: Center for Radiophysics and Space Research, Cornell University, Ithaca, NY 14853

${ }^{\dagger}$ Also at the Department of Astronomy and NCSA, University of Illinois at Urbana-Champaign, Urbana, IL 61801
}

There are at least two distinct effects which amplify the magnetic field in a HMNS: magnetic winding and the magnetorotational instability (MRI) [18, 19]. Numerical simulations using a general relativistic magnetohydrodynamics (GRMHD) code are required to follow this growth and determine the consequences. The key subtlety is that the wavelength of the fastest-growing MRI mode must be well resolved on the computational grid. Since this wavelength is proportional to the magnetic field strength, it becomes very difficult to resolve for small seed fields. However, the simulations reported here succeed in resolving MRI.

New computational tools now make long-term numerical evolutions of relativistic magnetized HMNSs possible for the first time. Two groups have independently developed codes for evolving MHD fluids in strong-field, dynamical spacetimes [7, [8] (see also [9]). These codes solve the Einstein-Maxwell-MHD system of coupled equations, both in axisymmetry and in 3+1 dimensions, essentially without approximation. Both codes evolve the spacetime metric using the BSSN formulation [10] and employ conservative shock-capturing schemes to integrate the GRMHD equations. Multiple tests have been performed with these codes, including MHD shocks, MHD wave propagation, magnetized Bondi accretion, MHD waves induced by gravitational waves, and magnetized accretion onto a neutron star.

To study the effects of magnetic fields on HMNSs, we first construct initial data assuming a $\Gamma=2$ polytropic EOS, $P=K \rho^{\Gamma}$, where $P, K$, and $\rho$ are the pressure, polytropic constant, and rest-mass density. Henceforth, we adopt units such that $K=c=G=1$, where $c$ is the speed of light and $G$ is the gravitational constant. In these units, the maximum allowed baryon masses $M_{0}$ of nonrotating and of rigidly rotating stars are 0.180 and 0.207 , respectively [11].

Following previous papers (e.g, [1, 6, 12]), we choose the rotation law $u^{t} u_{\varphi}=A^{2}\left(\Omega_{c}-\Omega\right)$, where $u^{\mu}$ is the fourvelocity, $\Omega \equiv u^{\varphi} / u^{t}$ is the angular velocity, and $\Omega_{c}$ is the angular velocity at the rotation axis. The constant $A$ has units of length and determines the degree of differential 
rotation. In this paper, $A$ is set equal to the coordinate equatorial radius $R$, giving a value of $\sim 1 / 3$ for the ratio of equatorial to central $\Omega$.

We model a HMNS with $M_{0}=0.303$, maximum density $\rho_{\max }=0.0668$, and angular momentum parameter $J / M^{2}=1.00$ [13]. The Arnowitt-Deser-Misner (ADM) mass is given by $M=0.279$, which is about $70 \%$ larger than the OV limit. Because of rapid differential rotation, the shape of this star is highly flattened (see the first panel of Fig. 2).

During the evolution, we adopt a $\Gamma$-law (adiabatic) EOS $P=(\Gamma-1) \rho \varepsilon$ with $\Gamma=2$. Here, $\varepsilon$ denotes the specific internal energy. Before evolving, we add a weak poloidal magnetic field to the equilibrium model by introducing a vector potential of the form $A_{\varphi}=$ $\varpi^{2} \max \left[A_{b}\left(P-P_{\text {cut }}\right), 0\right]$, where $P_{\text {cut }}$ is $4 \%$ of the maximum pressure, $A_{b}$ is a constant that determines the initial strength of the magnetic field, and $\varpi$ is the cylindrical radius. (A similar form of vector potential has been used in other MHD simulations [14].) We characterize the strength of the initial magnetic field by $C \equiv \max \left(b^{2} / P\right)$, the maximum value on the grid of the ratio of the magnetic energy density $b^{2}$ to the pressure. Several values of $A_{b}$ are chosen to yield the following values of $C$ : $1.26 \times 10^{-3}, 2.47 \times 10^{-3}, 4.88 \times 10^{-3}$, and $9.80 \times 10^{-3}$. We have verified that these small initial magnetic fields introduce negligible violations of the Hamiltonian and momentum constraints. Note that $C \propto v_{A}^{2}$, where $v_{A}$ is the Alfvén speed. Thus, $C^{-1 / 2} \propto v_{A}^{-1}$ is proportional to the Alfven time $t_{A}=R / v_{A}$. If the evolution time scale is determined by the Alfvén time, a scaling relation in the evolution should hold. This relation may not hold for all $C$ due to MRI, which grows exponentially at a rate independent of $t_{A}$. Comparing simulations with the values of $C$ quoted above, we indeed find that, if the time is rescaled as $C^{-1 / 2} t$, the results for different values of $C$ are approximately the same. (Detailed results will be shown in [15].) We therefore focus on the case with $C=2.47 \times 10^{-3}$. The typical value of $P$ for our model is $\sim 10^{34} \mathrm{erg} / \mathrm{cm}^{3}\left(2.8 M_{\odot} / M\right)^{2}$; the initial maximum magnetic field strength is then $\sim 10^{16}\left(2.8 M_{\odot} / M\right)$ gauss at $t=0$. (These scalings with $M$ assume our adopted initial polytropic model, for which $M / R=0.22$ ). This magnetic field is too strong to model a typical HMNS (but is similar in strength to 'magnetar' fields [16]). However, the qualitative behavior obtained here still applies as long as the approximate scaling relation holds.

Simulations in axisymmetry were performed independently using two GRMHD codes [7, 8], and the numerical results are qualitatively similar. As in many hydrodynamic simulations, we add a tenuous "atmosphere" to cover the computational grid outside the star. The atmospheric rest-mass density is set to $10^{-7} \rho_{\max , 0}$ for the simulations shown here, where $\rho_{\max , 0}$ is the maximum value of $\rho$ at $t=0$, which is 0.0668 in the adopted units.

We perform simulations on a uniform grid with size $(N, N)$ in cylindrical coordinates $(\varpi, z)$, which covers the region $[0,4.5 R]$ for each direction. For the HMNS

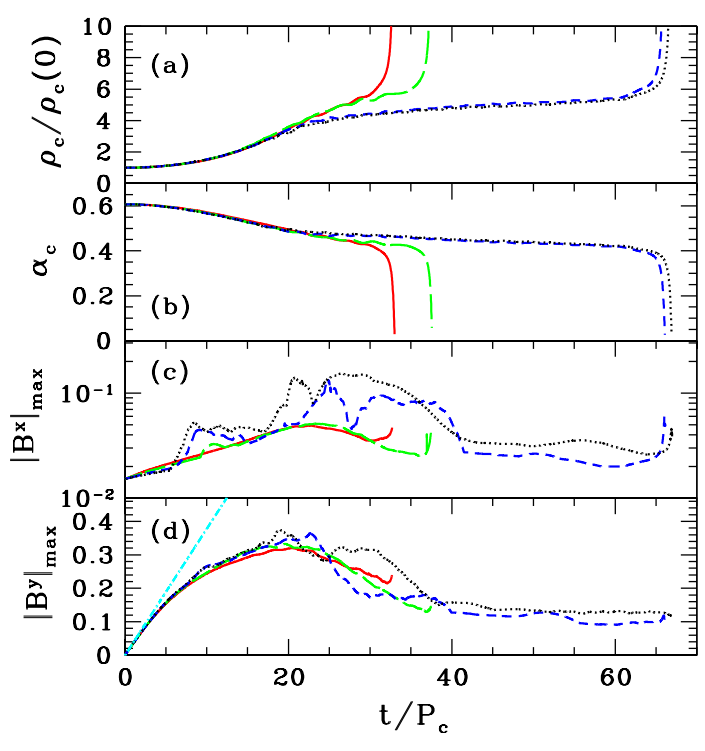

FIG. 1: Evolution of the central density, central lapse, and maximum values of $\left|B^{x}\right|$ and $\left|B^{y}\right|$ (the behavior of $\left|B^{z}\right|_{\max }$ is similar to the behavior of $\left|B^{x}\right|_{\max }$ and is therefore not shown). $\left|B^{x}\right|_{\max }$ and $\left|B^{y}\right|_{\max }$ are plotted in units of $\sqrt{\rho_{\max , 0}}$ where $\rho_{\max , 0}$ is the maximum rest-mass density at $t=0$. The solid (red), long-dashed (green), dashed (blue), and dotted (black) curves denote the results with $N=250,300,400$, and 500 respectively. The dot-dashed (cyan) line in the last panel represents the predicted linear growth of $B^{y}$ at early times.

adopted here, $R \approx 4.5 M \approx 18.6 \mathrm{~km}\left(M / 2.8 M_{\odot}\right)$. To check the convergence of our numerical results, we perform simulations with four different grid resolution: $N=250,300,400$ and 500 . We also checked that moving the outer boundary between $4 R$ and $5 R$ does not significantly affect the results.

Fig. 1 shows the evolution of the central density $\rho_{c}$, central lapse $\alpha_{c}$, and the maximum values of $\left|B^{x}\right|(\equiv$ $\left.\left|B^{\varpi}\right|\right)$ and $\left|B^{y}\right|\left(\equiv \varpi\left|B^{\varphi}\right|\right)$ as functions of $t / P_{c}$. Here $P_{c} \approx 39 M=0.54\left(M / 2.8 M_{\odot}\right) \mathrm{ms}$ denotes the central rotation period at $t=0$. The central density monotonically increases with time up to the formation of a black hole. Evolutions with various grid resolutions demonstrate that the results begin to converge when $N \gtrsim 400$. On the other hand, results are far from convergent for $N \lesssim 300$. For example, the maximum values of $\left|B^{x}\right|$ are much smaller than those with higher resolutions, and the growth rate of $\left|B^{x}\right|$ is underestimated. Hence, the effect of MRI, which is responsible for the growth of $\left|B^{x}\right|$, is not computed accurately for low resolutions. This is because the wavelength of the fastest growing MRI mode is not well-resolved for low resolutions (see below).

Simulations with different treatments of the atmosphere are qualitatively the same when the atmospheric density is sufficiently small. However, the exact collapse time is somewhat sensitive to the details of numerical and atmospheric schemes. This is not surprising because, at late times, the star becomes marginally unstable, and 

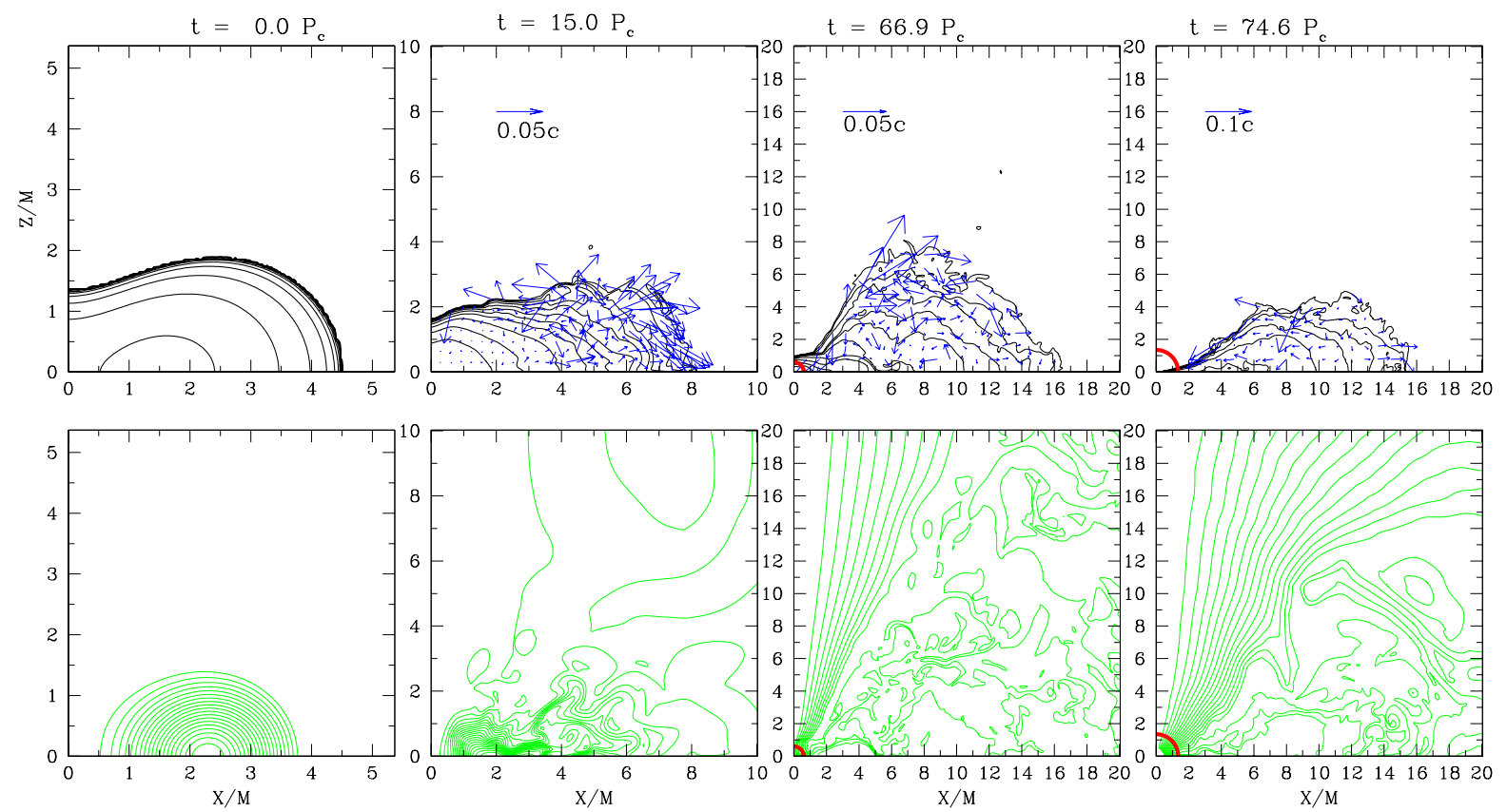

FIG. 2: The upper 4 panels show snapshots of the rest-mass density contours and velocity vectors on the meridional plane. The lower panels show the field lines (lines of constant $A_{\phi}$ ) for the poloidal magnetic field at the same times as the upper panels. The density contours are drawn for $\rho / \rho_{\max , 0}=10^{-0.3 i-0.09}(i=0-12)$. The field lines are drawn for $A_{\phi}=A_{\phi, \min }+\left(A_{\phi, \max }-\right.$ $\left.A_{\phi, \text { min }}\right) i / 20(i=1-19)$, where $A_{\phi, \text { max }}$ and $A_{\phi, \text { min }}$ are the maximum and minimum value of $A_{\phi}$ respectively at the given time. The thick solid (red) curves denote the apparent horizon.

the precise onset of collapse over the secular lifetime is sensitive to small differences in different schemes.

For the chosen initial strength of the seed magnetic field, the early evolution is dominated by magnetic winding. When the seed field is weak, the induction equation shows that $B^{y}$ grows approximately linearly: $B^{y}(t ; \varpi, z) \approx t \varpi B^{i}(0 ; \varpi, z) \partial_{i} \Omega(0 ; \varpi, z)$. Indeed, the early growth rate agrees with the predicted one (cf. dotdashed line in the last panel of Fig. 11). When the energy stored in the toroidal field becomes significant compared to the rotational energy, $\left|B^{y}\right|$ grows more slowly and the degree of differential rotation is reduced. Eventually $\left|B^{y}\right|$ reaches a maximum and starts to decrease. This is expected to happen on the Alfvén time scale $t_{A}$ [17], where the Alfvén speed is $v_{A}=\sqrt{b^{2} /\left(\rho h+b^{2}\right)}$. For the model considered here, the maximum value of $v_{A}=0.00734$, and thus, the minimum value of $t_{A}=15.8 P_{c}$. We see that the maximum value of $\left|B^{y}\right|$ starts decreasing when $t \gtrsim 20 P_{c}$, consistent with the Alfvén time scale.

MRI is evident at times $t \lesssim 6 P_{c}$ as shown in Fig. [1 where the maximum value of $\left|B^{x}\right|$ suddenly increases rapidly. MRI occurs wherever $\partial_{\varpi} \Omega<0$ [19]. The wavelength for the fastest growing mode is $\lambda_{\mathrm{MRI}} \approx$ $2 \pi v_{A} / \Omega$ and the e-folding time of the growth is $\tau_{\mathrm{MRI}}=$ $2(\partial \Omega / \partial \ln \varpi)^{-1}[19]$. With our choice of the initial magnetic field strength, $\lambda_{\mathrm{MRI}} \sim R / 10$ and $\tau_{\mathrm{MRI}} \sim 1 P_{c}$. In Fig. [1 we see that MRI shows up prominently when $N \gtrsim$ 400. Hence, we need to use a resolution $\Delta \lesssim \lambda_{\mathrm{MRI}} / 10$ to study the effect of MRI accurately. We find that MRI first occurs in the outer layers of the star near the equatorial plane. This can also be seen in Fig. 2] where we see that the magnetic field lines are distorted by $t=15 P_{c}$. In Fig. 10 we see that the the central density begins to grow more slowly once $\left|B^{x}\right|$ saturates. This may be caused by MRI-induced turbulence redistributing some of the angular momentum to slow down the contraction of the core.

The combined effects of magnetic braking due to winding and MRI eventually trigger gravitational collapse to a black hole at $t \approx 66 P_{c} \approx 36\left(M / 2.8 M_{\odot}\right)$ ms when an apparent horizon forms. The latest simulations [5] of BNS mergers show that for a sufficiently stiff EOS and typical observed BNS masses, HMNS formation is possible. HMNS remnants are triaxial and strong emitters of gravitational waves in these simulations. The dissipation time scale of angular momentum due to gravitational radiation is $\sim 100 \mathrm{~ms}[5]$. Therefore, HMNSs with an initially large magnetic field $\left(B \gtrsim 10^{16} \mathrm{G}\right)$ will be subject to 'delayed' collapse due to MHD effects (magnetic braking + MRI) rather than by the emission of gravitational waves. For seed magnetic fields which are much weaker than the cases studied here, gravitational radiation may be the trigger of collapse. However, it is possible that MRI may dominate the evolution even in this case, since the e-folding time of MRI is independent of the initial field strength. A more careful study of this scenario has 
to be carried out in full 3D [20]. However, since any dissipative agent (viscosity, magnetic fields, gravitational radiation) serves to redistribute and/or carry off angular momentum, the final fate of an HMNS - collapse to a black hole, accompanied by a gravitational wave burst is assured.

Soon after the formation of the apparent horizon, the simulations become inaccurate because of grid stretching. To follow the subsequent evolution, a simple excision technique for black hole spacetimes is employed [21, 22]. The evolution of the irreducible mass of the black hole computed from the area of the apparent horizon $A_{\mathrm{AH}}$ as $M_{\mathrm{irr}}=\sqrt{A_{\mathrm{AH}} / 16 \pi}$, and the total rest mass outside the apparent horizon are shown in Fig. 3. Soon after formation, the black hole grows rapidly, swallowing the surrounding matter. However, the accretion rate $\dot{M}_{0}$ gradually decreases and the black hole settles down to a quasiequilibrium state, i.e., the growth time scale becomes much longer than the dynamical time scale. At the end of the simulation, $\dot{M}_{0}$ decreases to $\approx 0.01 M_{0} / P_{c}$. The estimated value of the black hole spin parameter is $J_{\text {hole }} / M_{\text {hole }}^{2} \sim 0.8$. The black hole angular momentum is computed from $J_{\text {hole }}=J-J_{\text {matter }}\left(r>r_{H}\right)$, where the angular momentum of the matter outside the horizon $J_{\text {matter }}\left(r>r_{H}\right)$ is computed by a volume integral (see e.g., Eq. (51) of [6]). The mass of the black hole $M_{\text {hole }}$ is crudely estimated from $M_{\text {hole }} \approx \sqrt{M_{\text {irr }}^{2}+\left(J_{\text {hole }} / 2 M_{\mathrm{irr}}\right)^{2}}$. The density contour curves and magnetic field lines at the end of the simulation are shown in the last column of panels of Fig. [2

The value of $M_{0}$ indicates that the accretion time scale is $\sim 10-20 P_{c} \approx 5-10 \mathrm{~ms}\left(M / 2.8 M_{\odot}\right)$. Also, we find that the specific internal thermal energy in the torus near the surface is substantial because of shock heating, indicating that the torus can be a strong emitter of neutrinos.
These facts suggest that the system formed after the 'delayed' collapse of a magnetized HMNS (black hole + hot torus + collimated magnetic field) is a candidate for the central engine of short gamma-ray bursts 23, 24]. This possibility is explored in more detail in [25].

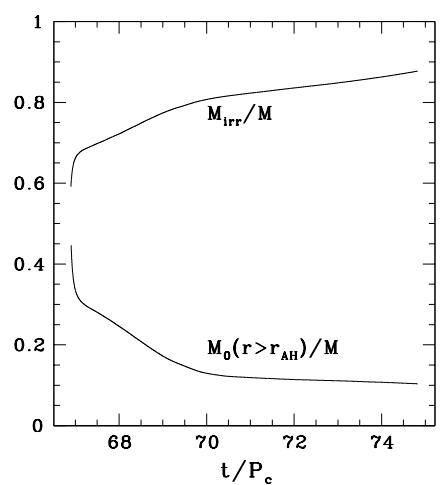

FIG. 3: Evolution of the irreducible mass and the total restmass outside the apparent horizon.

\section{Acknowledgments}

It is a pleasure to thank C. Gammie for useful suggestions and discussions. Numerical computations were performed at the National Center for Supercomputing Applications at the University of Illinois at UrbanaChampaign (UIUC), and on the FACOM VPP5000 machine at the data analysis center of NAOJ and the NEC SX6 machine in ISAS, JAXA. This work was in part supported by NSF Grants PHY-0205155 and PHY-0345151, NASA Grants NNG04GK54G and NNG046N90H at UIUC, and Japanese Monbukagakusho Grants (Nos. 17030004 and 17540232).
[1] T. W. Baumgarte, S. L. Shapiro, and M. Shibata, Astrophys. J. Lett. 528, L29 (2000).

[2] F. A. Rasio and S. L. Shapiro, Astrophys. J. 432, 242 (1994); Class. Quant. Grav. 16 R1 (1999).

[3] J. A. Faber and F. A. Rasio, Phys. Rev. D 62, 064012 (2000).

[4] M. Shibata, K. Taniguchi, and K. Uryū, Phys. Rev. D 68, 084020 (2003).

[5] M. Shibata, K. Taniguchi, and K. Uryū, Phys. Rev. D 71, 084021 (2005).

[6] M. D. Duez, Y. T. Liu, S. L. Shapiro, and B. C. Stephens, Phys. Rev. D 69, 104030 (2004).

[7] M. D. Duez, Y. T. Liu, S. L. Shapiro, and B. C. Stephens, Phys. Rev. D 72, 024028 (2005).

[8] M. Shibata and Y.-I. Sekiguchi, Phys. Rev. D 72, 044014 (2005).

[9] L. Antón, O. Zanotti, J. A. Miralles, J. M. Martí, J. M. Ibáñez, J. A. Font, and J. A. Pons, Astrophys. J., in press, astro-ph/0506063 (2005).

[10] M. Shibata and T. Nakamura, Phys. Rev. D 52, 5428 (1995); T. W. Baumgarte and S. L. Shapiro, Phys. Rev.
D 59, 024007 (1999).

[11] G. C. Cook, S. L. Shapiro, and S. A. Teukolsky, Astrophys. J. 422, 227 (1994).

[12] M. Shibata, T. W. Baumgarte, and S. L. Shapiro, Astrophys. J. 542, 453 (2000).

[13] The star chosen here is the same as that referred to as star I in [6].

[14] J-P. De Villiers, J. F. Hawley, and J. H. Krolik, Astrophys. J., 599, 1238 (2003); J. C. McKinney and C. F. Gammie, Astrophys. J., 611, 977 (2004)

[15] M. D. Duez, Y. T. Liu, S. L. Shapiro, M. Shibata, and B. C. Stephens, in preparation.

[16] R. C. Duncan and C. Thompson, Astrophys. J. Lett., 392, 9 (1992).

[17] S. L. Shapiro, Astrophys. J. 544, 397 (2000); J. N. Cook, S. L. Shapiro, and B. C. Stephens, Astrophys. J. 599, 1272 (2003).

[18] V. P. Velikhov, Soc. Phys. JETP, 36, 995 (1959); S. Chandrasekhar, Proc. Natl. Acad. Sci. USA, 46, 253 (1960).

[19] S. A. Balbus and J. F. Hawley, Astrophys. J. 376, 214 
(1991); Rev. Mod. Phys. 70, 1 (1998).

[20] The growth of MRI may be significantly enhanced when axisymmetry is relaxed; see J. F. Hawley, Astrophys. J. 528, 462 (2000).

[21] M. Alcubierre and B. Brügmann, Phys. Rev. D 63, 104006 (2001).

[22] M. D. Duez, S. L. Shapiro, and H-J. Yo, Phys. Rev. D 69, 104016 (2004).
[23] E.g., T. Piran, Phys. Rep. 314, 575 (1999); ibid 333, 529 (2000): Rev. Mod. Phys. 76, 1143 (2005).

[24] M. A. Aloy, H.-T. Janka, and E. Müller, Astron. Astrophys. 436, 273 (2005)

[25] M. Shibata, M. D. Duez, Y. T. Liu, S. L. Shapiro, and B. C. Stephens, submitted to Phys. Rev. Lett. (astro-ph/0511142). 